\title{
Perfusão regional do membro com antimicrobianos em equinos
}

\author{
Regional limb perfusion with antimicrobials in horses \\ Jackson Schade $^{1^{*}}$, Juliana Massitel Curti ${ }^{2}$, Gustavo Romero Gonçalves ${ }^{2}$, Taise Pissinato da Silva ${ }^{3}$ e \\ Emanuel Vitor Pereira Appolonio 4
}

\author{
${ }^{1}$ Universidade Positivo, Curitiba, PR, Brasil. *Autor para correspondência: schadejackson@yahoo.com.br. \\ ${ }^{2}$ Centro Universitário Ingá, Maringá, PR, Brasil. \\ 3Universidade Estadual de Londrina, Londrina, PR, Brasil. \\ ${ }^{4}$ Universidade Estadual Paulista, Botucatu, SP. Brasil.
}

Submissão: 19/03/2017 / Aceite: 25/02/2019

\begin{abstract}
RESUMO
As infecções ortopédicas são condições comumente observadas em equinos, afetando frequentemente estruturas nobres como ossos e tecido sinovial. Afecções de origem séptica são de extrema importância na espécie equina, pois podem resultar em claudicação permanente provocada por danos articulares irreversíveis. Desta forma, tratamentos que resultam em concentrações elevadas de antimicrobianos nas áreas infectadas atuam de maneira mais eficaz na eliminação da população bacteriana nestes tecidos. Neste sentido, a perfusão regional do membro tem se mostrado uma excelente alternativa. A presente revisão aborda os principais aspectos relacionados às técnicas de perfusão regional do membro com antimicrobianos em equinos. Serão descritas as técnicas intravenosa e intraóssea, as quais envolvem a administração de antimicrobianos, em uma região selecionada do membro, isolada previamente da circulação sistêmica por meio da aplicação de um torniquete. A perfusão regional tem sido descrita com sucesso no tratamento de condições sépticas da região distal do membro de equinos, visto que altas concentrações de antimicrobianos são alcançadas nos locais afetados. Além disso, é de fácil realização e apresenta baixo risco de efeitos adversos sistêmicos, bem como custo reduzido.
\end{abstract}

PALAVRAS-CHAVE: equino, infecções ortopédicas, torniquete.

\begin{abstract}
Orthopedic infections are conditions commonly observed in horses, often affecting important structures such as bone and synovial tissue. Septic source conditions are extremely important in horses as they may result in permanent lameness caused by irreversible joint lesions. Therefore, treatments that result in higher concentrations of antimicrobials in infected areas act more effectively eliminating the bacterial population in these tissues. Therefore, the regional limb perfusion has proven to be an excellent alternative. This review addresses the main issues of regional limb perfusion with antimicrobials in horses. It will describe intravenous and intraosseous techniques, which involve the administration of an antimicrobial solution, in a part of the limb, previously isolated from the systemic circulation by applying a tourniquet. Regional perfusion has been described as a successful treatment for septic conditions at the distal region of the equine limb, since high concentrations of antimicrobial are achieved at the sites affected. In addition, it is easy to perform and presents a low risk of adverse systemic effects as well as reduced cost.
\end{abstract}

KEYWORDS: equine, orthopedic infections, tourniquet.

\section{INTRODUÇÃO}

Infecções ortopédicas são condições comumente observadas em equinos (CIMETTI et al. 2004, RUBIO-MARTÍNEZ \& CRUZ 2006), as quais podem afetar estruturas nobres como ossos e tecido sinovial (RICHARDSON \& AHERN 2012). Estas estruturas são frequentemente colonizadas por bactérias como resultado de ferimentos traumáticos, cirurgia, injeção intra-articular (iatrogênica) ou pela disseminação hematógena (SCHNEIDER et al. 1992, RICHARDSON \& AHERN 2012).

$\mathrm{Na}$ espécie equina as infecções musculoesqueléticas são especialmente importantes, pois podem levar a claudicação permanente provocada por danos articulares irreversíveis, mesmo após a resolução da 
infecção (SCHNEIDER 2006). Sendo assim, o tratamento precoce deve ser instituído para que o retorno a atividade atlética seja possível (SCHNEIDER et al. 1992). Dentre outros aspectos, para o sucesso do tratamento, altas concentrações do antimicrobiano devem alcançar as áreas infectadas para efetiva eliminação da população bacteriana (RUBIO-MARTíNEZ \& CRUZ 2006). No entanto, com o comprometimento vascular resultante do processo, as bactérias são protegidas dos mecanismos de defesa do hospedeiro e da ação dos antimicrobianos administrados por via sistêmica (MATTSON et al. 2004). Nesse caso, a perfusão regional do membro (PRM) resulta em concentrações superiores de antimicrobianos em tecidos pouco perfundidos da extremidade distal quando comparada com administração sistêmica (MURPHEY et al. 1999, PILLE et al. 2005).

O objetivo do trabalho foi abordar os principais aspectos relacionados à utilização das modalidades intravenosa e intraóssea de perfusão regional do membro com antimicrobianos, fornecendo um guia para a realização da técnica no tratamento das infecções ortopédicas dos equinos.

\section{DESENVOLVIMENTO}

\section{Modalidades}

Duas modalidades utilizadas para PRM com antimicrobianos são descritas: intravenosa (PRMIV) e intraóssea (PRMIO) (BUTT et al. 2001, RUBIO-MARTÍNEZ et al. 2005, RICHARDSON \& AHERN 2012). Ambas envolvem a administração de uma solução em uma região selecionada do membro, isolada previamente da circulação sistêmica por meio da aplicação controlada de um torniquete (BUTT et al. 2001, RUBIO-MARTÍNEZ et al. 2005). Portanto, técnicas de perfusão regional são exclusivamente realizadas na região distal dos membros (distal ao terço médio do rádio e tíbia), devido a impossibilidade de isolar regiões da região proximal (RUBIO-MARTÍNEZ \& CRUZ 2006).

Em ambas as modalidades (PRMIV ou PRMIO), o procedimento pode ser realizado com o equino em estação com ou sem sedação, ou em decúbito sob anestesia geral (RUBIO-MARTíNEZ \& CRUZ 2006, LUGO 2009). Previamente à perfusão, um torniquete deve ser aplicado proximal ao local de infecção e ao acesso vascular (SANTSCHI et al. 1998, BUTT et al. 2001) com objetivo de ocluir os sistemas arterial e venoso, inibindo a chegada e a saída de sangue e antimicrobiano do local isolado para a circulação sistêmica (MATTSON et al. 2004, RUBIO-MARTíNEZ \& CRUZ 2006) (Figura 1).

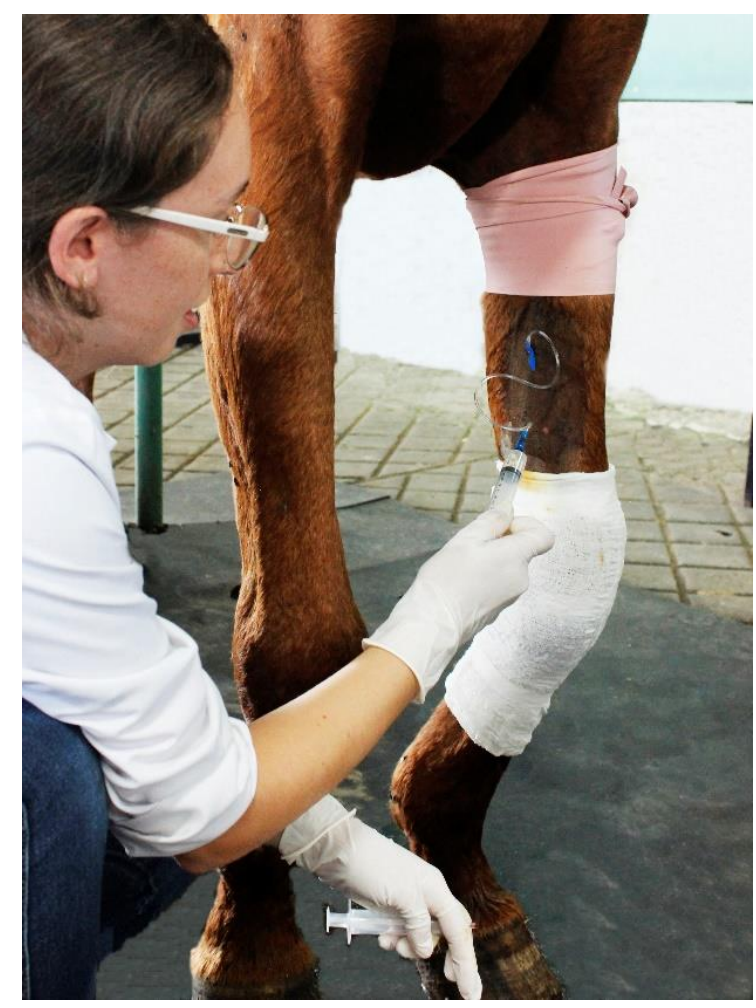

Figura 1. Perfusão regional do membro com antimicrobiano realizada com o equino em estação. $O$ garrote foi aplicado no terço médio do antebraço, proximal ao local da infecção e ao acesso vascular.

Figure 1. Regional limb Perfusion with antimicrobial performed with horse in standing position. The tourniquet was applied to the middle third of the forearm, proximal to the infection site and to vascular access 
Devido ao desconforto gerado pela aplicação do torniquete, a realização de sedação e bloqueios anestésicos perineurais proximal ao local de garroteamento torna-se necessária (RUBIO-MARTíNEZ \& CRUZ 2006, RUBIO-MARTíNEZ et al. 2012), visto que a movimentação do membro poderá contribuir para a perda do antimicrobiano, sob o torniquete, para a circulação sistêmica (LUGO 2015). O desconforto causado pelo torniquete poderia ainda ser reduzido por meio da infusão de $10 \mathrm{~mL}$ de anestésico local como a mepivacaína $2 \%$ ou lidocaína $2 \%$ sem vasoconstritor, adicionada ao volume final da solução administrada (RUBIO-MARTíNEZ et al. 2012, KELMER et al. 2014, RAFAEL et al. 2014).

Para a PRMIV, qualquer veia superficial visível e acessível, distal ao torniquete, poderá ser utilizada para administração da solução antibiótica. Frequentemente, para as regiões distais ao metacarpo e metatarso, as veias digitais são utilizadas (PALMER \& HOGAN 1999, LUGO 2009). As veias cefálica e safena poderão ser empregadas para perfusão da região distal do terço distal do rádio e tíbia, respectivamente, incluindo o carpo e o tarso (LUGO 2009) (Figura 2). No entanto, como alternativa a utilização das veias digitais, veias proximais, como a cefálica e safena, podem ser utilizadas para 0 tratamento de infecções da extremidade distal do membro (KELMER et al. 2012a, KELMER et al. 2012b). A via arterial não é recomendada, pois é associada à lesão endotelial severa, visto que os efeitos tóxicos sobre o endotélio são mais frequentes e graves nas artérias que nas veias (RUBIO-MARTINEZ et al. 2006).

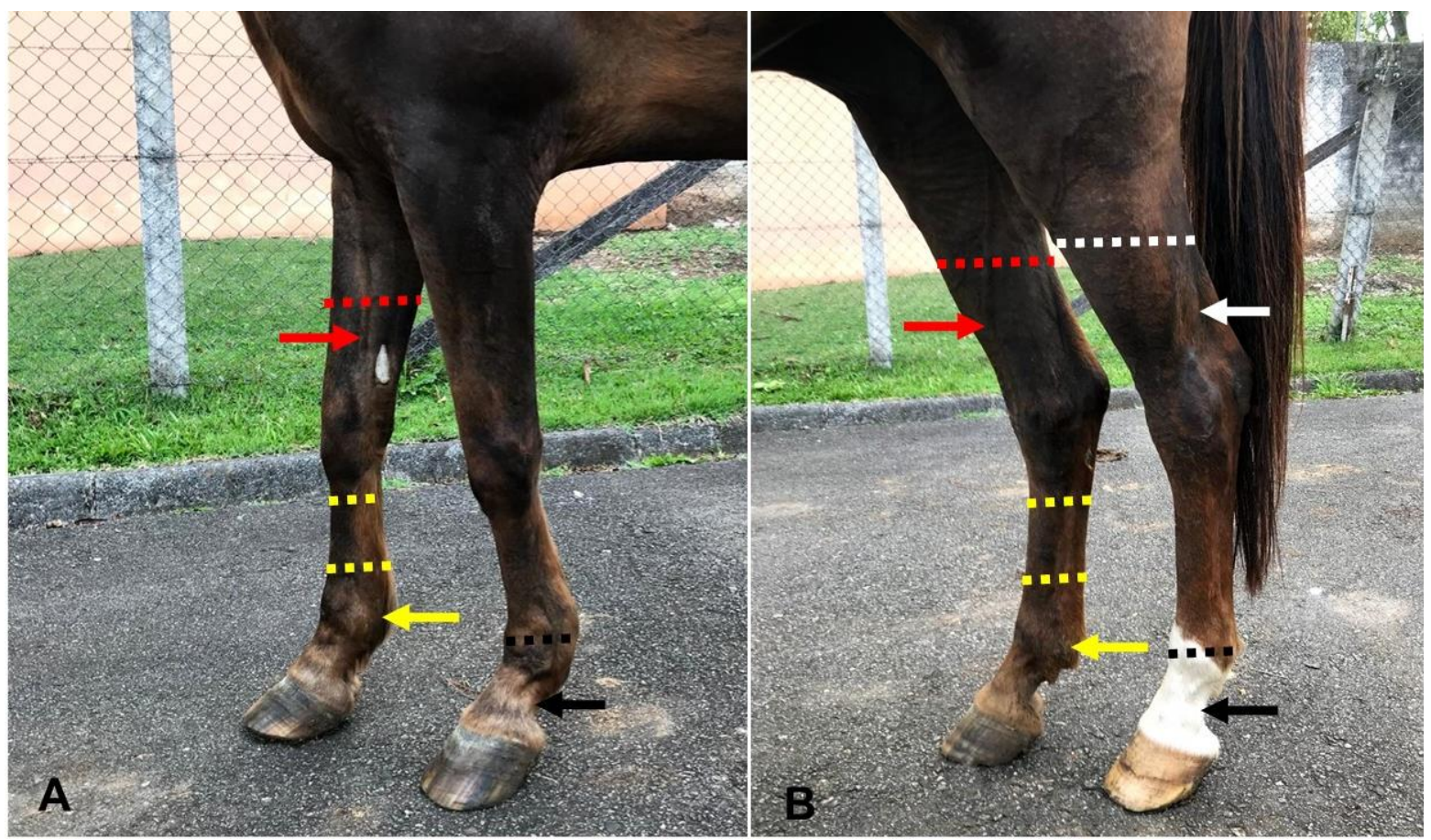

Figura 2. Locais de acesso venoso mais comumente utilizados para perfusão regional com antimicrobianos em equinos (setas) e local de aplicação do torniquete correspondente ao vaso utilizado (linha pontilhada da mesma cor da seta). Veia cefálica (seta vermelha) (A), veia safena medial (seta vermelha), veia safena lateral (seta branca) (B), veias digitais (seta amarela e preta) (A e B).

Figure 2. Sites of venous access most commonly used for regional perfusion with antimicrobial agents in horses (arrows) and place of application of the tourniquet corresponding to the vessel used (dotted line of the same arrow color). Cephalic vein (red arrows) (A), medial saphenous vein (red arrows), lateral saphenous vein (white arrow) (B) and digital veins (yellow and black arrow) ( $A$ and $B)$.

Após antissepsia, o acesso venoso poderá ser realizado por meio da utilização de agulhas do tipo "butterfly" (scalp) ou cateter venoso periférico, geralmente 18-20 gauge para equinos adultos e 22 gauge para potros (RUBIO-MARTíNEZ \& CRUZ 2006). Antissepsia cirúrgica é justificada em locais próximos a estruturas sinoviais, como as veias digitais próximas ao recesso proximal da bainha digital e a veia safena medial dorsalmente a articulação tarsocrural (SISSON \& GROSSMAN 1986), devido ao risco de punção sinovial inadvertida. Quando repetidas perfusões são necessárias, os dispositivos intravenosos de menor calibre são mais adequados, visto que causam menor dano ao vaso (SANTSCHI et al. 1998, RUBIOMARTÍNEZ \& CRUZ 2006). Em estudo realizado por KELMER et al. (2009), os autores demonstraram que a manutenção de cateteres de poliuretano 20 gauge nas veias safena, cefálica e digitais foi possível por até sete dias, com mínimas complicações, constituindo uma alternativa quando repetidas perfusões são 
necessárias. No entanto, RUBIO-MARTÍNEZ et al. (2012), em estudo retrospectivo de casos, observaram menor frequência de complicações $(12,26 \%)$ ao utilizarem agulhas do tipo "butterfly" 22 gauge, quando comparados com os resultados de KELMER et al. (2012b), os quais observaram complicações em $27 \%$ dos membros ao utilizarem cateteres de politetrafluoroetileno 20 gauge ou poliuretano 16 gauge, implantados e fixados nas veias safena e cefálica.

A técnica de PRMIO consiste na injeção de uma solução de fármacos diretamente na cavidade medular do osso, precedida pela aplicação controlada de um torniquete (BUTT et al. 2001, WERNER et al. 2003, MATTSON et al. 2004). Para realização desta técnica, a introdução de um parafuso ósseo cortical, especialmente projetado, ligado a um conector (tipo Luer-luck) poderá ser efetuada, por meio do qual será infundida a solução (MATTSON et al. 2004, RUBIO-MARTINEZ et al. 2005, CRUZ et al. 2006). O parafuso é implantado cirurgicamente na cavidade medular do metacarpo, metatarso, rádio ou tíbia (CRUZ et al. 2006), sem a necessidade de anestesia geral para realização do procedimento. No entanto, a utilização de bloqueios anestésicos perineurais e/ou anestesia infiltrativa local é geralmente necessária como método de analgesia (LUGO 2009). De acordo com a técnica descrita por MATTSON et al. (2004), após preparação cirúrgica asséptica, uma incisão de aproximadamente $1,0 \mathrm{~cm}$ é realizada através da pele, tecido subcutâneo e periósteo. O tecido mole é cuidadosamente retraído, seguido pela perfuração de um orifício de $4 \mathrm{~mm}$ de diâmetro através da cortical, no qual um parafuso de $5,5 \mathrm{~mm} \times 20 \mathrm{~mm}$ é introduzido. Após completar a perfusão intraóssea, recomenda-se a injeção de solução salina estéril $(\mathrm{NaCl} 0,9 \%$ ) heparinizada no parafuso canulado, seguido pela confecção e manutenção de bandagem no membro para a proteção do parafuso, o qual permanecerá implantado (RUBIO-MARTíNEZ et al. 2012).

Em ambas as técnicas (PRMIV ou PRMIO), a perfusão da solução poderá ser precedida (PILLE et al. 2005, KELMER et al. 2009) ou não (PALMER \& HOGAN 1999, LEVINE et al. 2010) por exsanguinação do membro. Entretanto, quando não realizada, um grande volume de perfusão poderia diminuir a eficácia do torniquete devido à pressão intravascular excessiva, justificando a redução do volume total da solução (RUBIO-MARTINÉZ \& CRUZ 2006). A remoção do sangue pode ser realizada por meio da aplicação de faixas Esmarch no sentido distal para proximal (MURPHEY et al. 1999, RUBIO-MARTíNEZ et al. 2005) ou pela aspiração de volume de sangue equivalente ao da solução de perfusão (KELMER et al. 2009). Quando a via intravenosa é utilizada, recomenda-se que o acesso venoso seja realizado antes da exsanguinação, pois após o procedimento o colapso venoso resultante dificultará o acesso ao vaso (NAVARRE et al. 1999, RUBIO-MARTÍNEZ \& CRUZ 2006).

A escolha da técnica de perfusão regional do membro é baseada principalmente na disponibilidade do equipamento e na preferência do médico veterinário. No entanto, nos casos em que é impossível identificar o acesso vascular, a utilização da via intraóssea torna-se a mais indicada (BUTT et al. 2001, LUGO 2009). Complicações frequentemente associadas a PRMIV consistem em hematoma, trombose (RUBIOMARTíNEZ et al. 2012), flebite, edema de tecidos moles e dificuldade de acesso venoso, especialmente quando repetidas perfusões são necessárias (BUTT et al. 2001, MATTSON et al. 2004). Nesse sentido, a implantação de um parafuso intraósseo permite acesso para perfusão do membro, repetida por vários dias e com poucas complicações. Entretanto, constitui um procedimento mais invasivo, frequentemente associado a desconforto devido a elevada pressão intraóssea durante a perfusão (MATTSON et al. 2004, KELMER et al. 2009). Possíveis complicações associadas a PRMIO incluem drenagem ao redor do parafuso, dificuldade de injeção da solução, afrouxamento do parafuso (RUBIO-MARTíNEZ et al. 2012), quebra de brocas, parafusos ou fratura óssea iatrogênica (MATTSON et al. 2004) e osteonecrose (PARKER et al. 2010).

\section{Torniquetes}

A escolha e a correta aplicação do torniquete são parte fundamental na realização da PRM com antimicrobianos, visto que podem influenciar a concentração do fármaco na região de interesse (LEVINE et al. 2010, ALKABES et al. 2011). Um torniquete ou garrote consiste em uma faixa circunferencial, aplicada com pressão ao redor do membro, com o objetivo de comprimir os vasos sanguíneos (artérias e veias), inibindo o fluxo e o efluxo de sangue, promovendo isolamento vascular da região anatômica de interesse (ALKABES et al. 2011).

Vários são os fatores que influenciam a eficácia de um torniquete quando a PRM é realizada (RUBIOMARTÍNEZ \& CRUZ 2006). Durante a perfusão, a injeção da solução conduz ao aumento de pressão no compartimento venoso. Quando esta pressão alcança seu limite máximo ocorre vazamento da solução sob o torniquete através do sistema venoso. Dessa forma, fatores como pressão e largura do torniquete, volume da solução, local e taxa de infusão, exsanguinação prévia e características individuais do paciente são importantes determinantes da eficácia do torniquete (RUBIO-MARTINÉZ \& CRUZ 2006).

Os tipos de torniquetes utilizados em PRM incluem: torniquetes pneumáticos (Figuras 3A e 3B) 
(WERNER et al. 2003, KELMER et al. 2009), faixa Esmarch (CIMETTI et al. 2004, RUBIO-MARTíNEZ et al. 2005), torniquetes de borracha com diferentes larguras (BUTT et al. 2001, MATTSON et al. 2004) e torniquete cirúrgico tubular (MURPHEY et al. 1999) (Figura 4).
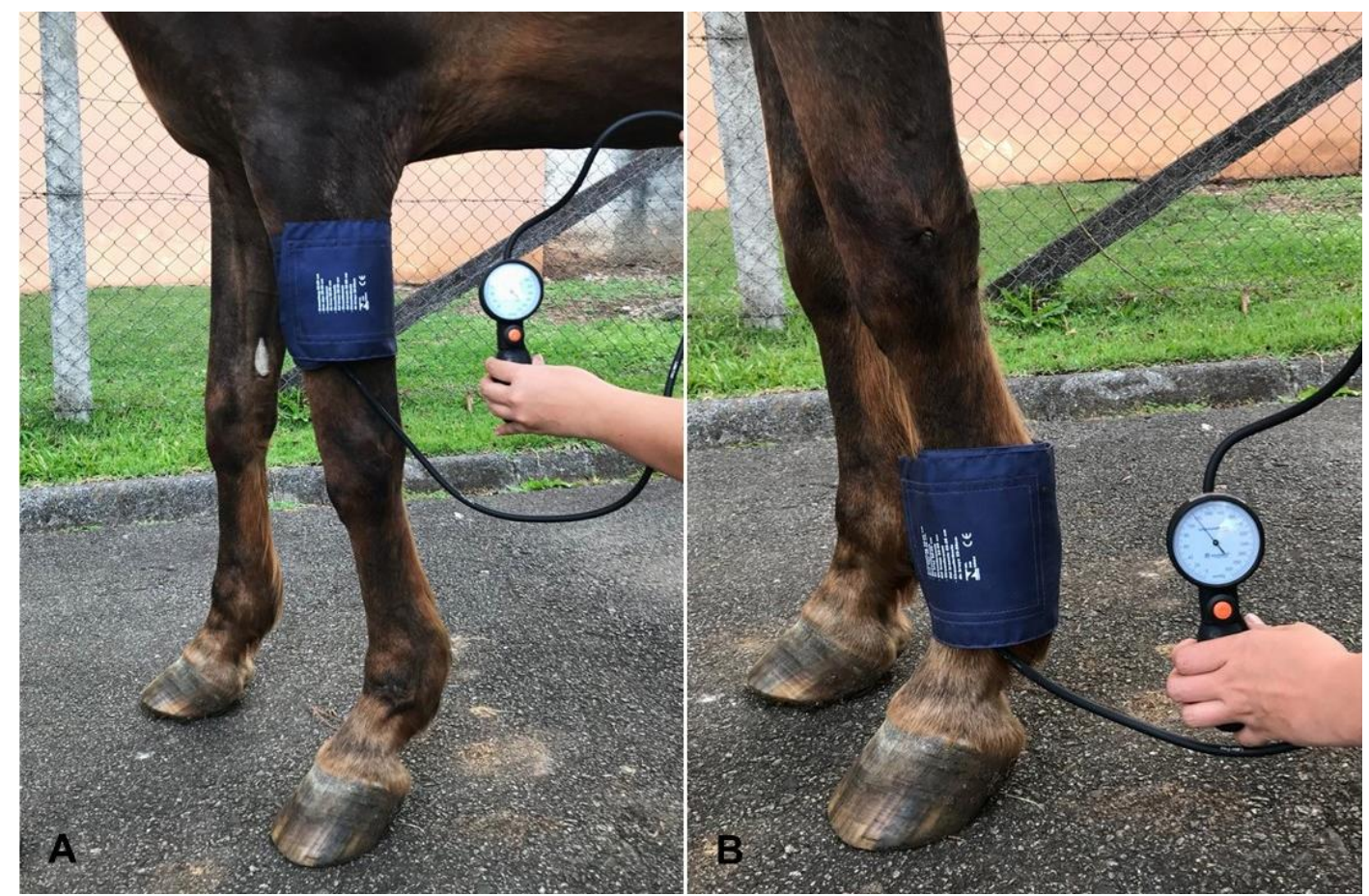

Figura 3. Imagem de um torniquete pneumático aplicado na região proximal (antebraço) e distal (boleto) do membro.

Figure 3. Image of a pneumatic tourniquet applied to the proximal (forearm) and distal (fetlock) regions of the limb.
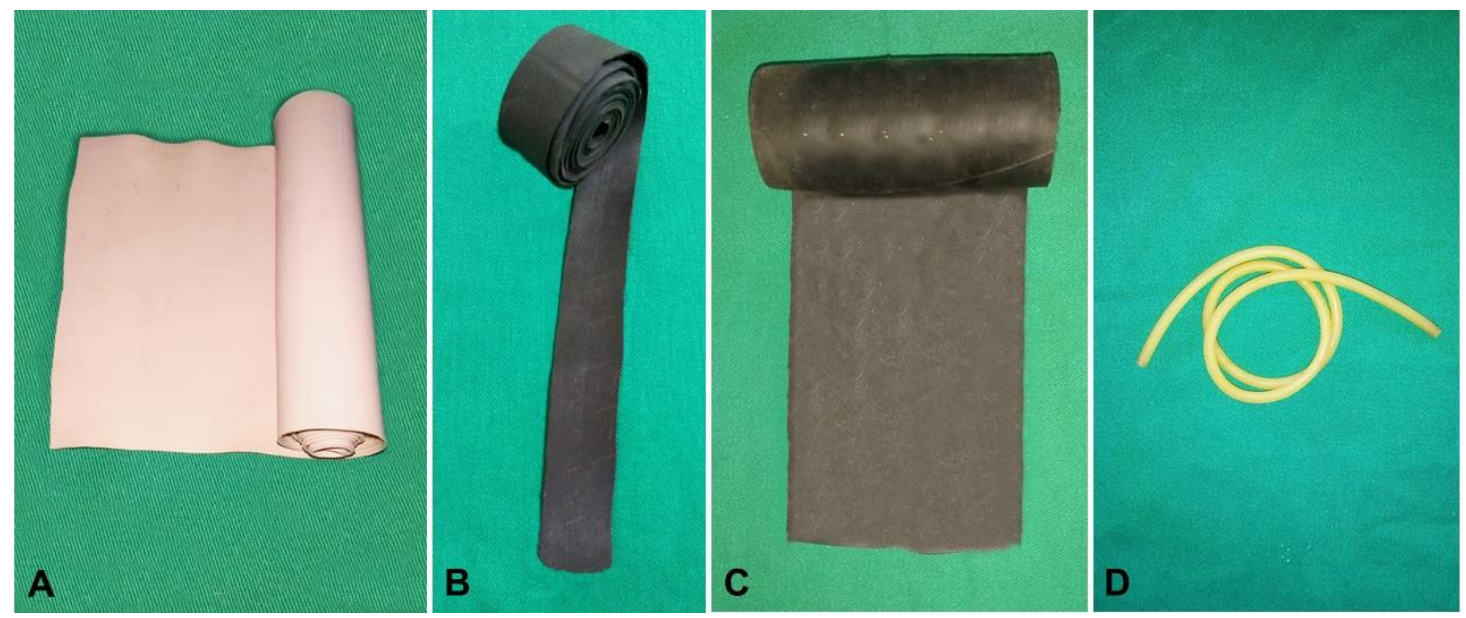

Figura 4. Imagem demonstrando os diferentes tipos de torniquetes. Faixa Esmarch $(A)$, torniquete fino (1,5 $\mathrm{cm}$ de largura) de borracha $(B)$, torniquete largo $(10 \mathrm{~cm})$ de borracha $(C)$ e torniquete cirúrgico tubular (D).

Figure 4. Image showing the different types of tourniquet. Esmarch band $(A)$, thin tourniquet $(1.5 \mathrm{~cm}$ of width), of rubber $(B)$, wide rubber tourniquet $(10 \mathrm{~cm})(C)$ and tubular surgical tourniquet $(D)$.

Torniquetes pneumáticos permitem o controle da pressão exercida no membro quando aplicado, o que não é possível quando outros tipos de torniquetes são utilizados (RUBIO-MARTíNEZ \& CRUZ 2006, ALKABES et al. 2011). A pressão ideal exercida pelo torniquete deve ser a mínima necessária para interromper o fluxo sanguíneo e foi definida como $100 \mathrm{mmHg}$ superior a pressão sistólica máxima, ou seja, cerca de 150-170 mmHg (ALKABES et al. 2011). No entanto, efeitos adversos não são observados quando valores de pressão superiores $(300-600 \mathrm{mmHg}$ ) são utilizados (WERNER et al. 2003, LEVINE et al. 2010, ALKABES et al. 2011). Em estudo realizado por ALKABES et al. (2011), o torniquete Esmarch teve maior 
eficácia quando comparado com o torniquete pneumático $(420 \mathrm{mmHg})$, além de ser mais barato e fácil de aplicar. LEVINE et al. (2010), observaram menor eficácia do torniquete de borracha mais estreito $(1 \mathrm{~cm})$ quando comparado com o torniquete de borracha mais largo $(12,5 \mathrm{~cm})$ e com o torniquete pneumático $(10,5 \mathrm{~cm}$ a $420 \mathrm{mmHg})$. Concentrações abaixo da concentração inibitória mínima (CIM) foram encontradas no líquido sinovial de equinos quando o torniquete de borracha estreita $(1 \mathrm{~cm})$ foi utilizado, o que, de acordo com o autor, desabona sua utilização para a PRM com antimicrobianos em equinos.

O tempo de 30 minutos para permanência do torniquete após completa infusão da solução é o mais amplamente utilizado e tem sido eficaz quando PRM com antimicrobianos é realizada (PILLE et al. 2005, KELMER et al. 2009, ALKABES et al. 2011). A duração mais adequada ainda não foi determinada (CRUZ et al. 2006), no entanto, características físico-químicas, farmacológicas e farmacodinâmicas do antimicrobiano, fatores microbiológicos, fatores individuais do paciente e do caso clínico poderiam influenciar o tempo de permanência do torniquete (RUBIO-MARTÍNEZ et al. 2005). Torniquetes mantidos por períodos de até duas horas induzem mínimos efeitos adversos sobre os equinos (RUBIO-MARTíNEZ \& CRUZ 2006). Hipertensão arterial sistêmica é o único efeito descrito em equinos adultos, a qual retorna ao intervalo normal de referência após remoção do torniquete (ABRAHAMSEN et al. 1989, COPLAND et al. 1989). No entanto, osteonecrose grave induzida pelo torniquete tem sido documentada em potros neonatos (RUBIOMARTÍNEZ \& CRUZ 2006).

\section{Seleção do antimicrobiano}

Pré-requisitos básicos como solubilidade em água e em soluções isotônicas balanceadas como $\mathrm{NaCl}$ $0,9 \%$ e Ringer com lactato são fundamentais na escolha do antimicrobiano mais adequado para a PRM (CRUZ et al. 2006, RUBIO-MARTÍNEZ \& CRUZ 2006). De maneira geral, a seleção do antimicrobiano deveria ser baseada em resultados de cultura e antibiograma de amostras coletadas da região infectada, antes do início do tratamento (SCHNEIDER et al. 1992). Entretanto, antes da obtenção dos resultados de suscetibilidade, a escolha do antimicrobiano pode ser realizada de acordo com os patógenos mais comumente associados com a infecção em questão (RUBIO-MARTíNEZ \& CRUZ 2006). Em estudo realizado por SCHNEIDER et al. (1992), os autores observaram que a etiologia da infecção tem influência significativa sobre os tipos de bactérias identificadas em culturas.

Os aminoglicosídeos, tais como a gentamicina e a amicacina, tem sido amplamente utilizados em estudos que avaliam perfusão regional do membro em equinos, demonstrando concentrações muito acima da CIM para a maioria dos patógenos nos tecidos alvo (MATTSON et al. 2004, LEVINE et al. 2010, ALKABES et al. 2011). Além da alta eficácia contra os patógenos comumente envolvidos em doenças ortopédicas (MOORE et al. 1992, LUGO 2009), o efeito bactericida, concentração dependente, dos aminoglicosídeos os torna antimicrobianos de escolha no tratamento de infecções ortopédicas por meio de PRM em equinos (LUGO 2009). MOORE et al. (1992) avaliando resultados de cultura e antibiograma de 233 equinos com infecções ortopédicas, observaram que a amicacina foi o antimicrobiano mais eficaz contra a maioria das bactérias isoladas, incluindo Gram negativas e Gram positivas. No entanto, resistência bacteriana a gentamicina tem sido demonstrada (MOORE et al. 1992).

Ascefalosporinas de terceira geração, tal como ceftiofur, apresentam amplo espectro de ação, incluindo organismos produtores de $\beta$-lactamases. Em estudo realizado por PILLE et al. (2005) utilizando PRM com ceftiofur, concentrações acima da CIM para os patógenos mais comuns foram observadas por mais de 24 horas no líquido sinovial da articulação antebraquiocárpica. Estes resultados demonstram que PRM com ceftiofur pode ser útil no tratamento de infecções ortopédicas em equinos. Entretanto, o isolamento de bactérias resistentes ao ceftiofur tem sido associado a uma nova classe de $\beta$-lactamases (PILLE et al. 2005).

Devido ao aumento da taxa de isolamento de microrganismos multirresistentes em equinos, tal como Staphylococcus spp. meticilina resistentes, antimicrobianos como a vancomicina tem sido estudado em equinos (HARTMANN et al. 1997). Avaliando a utilização de vancomicina por PRM em equinos, RUBIOMARTÍNEZ et al. (2005) observaram concentrações acima da CIM, durante os 90 minutos de estudo sem efeitos adversos, para Staphylococcus aureus meticilina resistente no plasma sinuisoidal da falange proximal. Este resultado sugere que a vancomicina pode representar uma possibilidade terapêutica para 0 tratamento de infecções ortopédicas em equinos. Entretanto, o autor ressalta que a vancomicina deve ser reservada para casos onde há resistência a outros antimicrobianos, após cultura e antibiograma, visto que o desenvolvimento de resistência bacteriana constituiria séria preocupação tanto para a saúde humana quanto animal.

Devido ao seu efeito vasculotóxico, a utilização de PRM com enrofloxacina vem sendo questionada (RUBIO-MARTÍNEZ \& CRUZ 2006). Em estudo realizado por PARRA-SANCHEZ et al. (2006), avaliando as 
características farmacocinéticas e farmacodinâmicas da enrofloxacina administrada por PRMIV em equinos, os autores evidenciaram vasculite em três dos sete equinos avaliados, sendo atribuído ao extravasamento da solução para o espaço perivascular. Os autores concluíram que a PRMIV com enrofloxacina pode ser considerada como opção de tratamento em infecções ortopédicas em equinos, no entanto, deve-se tomar cuidado para evitar o extravasamento do fármaco.

A combinação de dois antimicrobianos pode ser realizada quando as drogas apresentam efeitos sinérgicos, como é o caso dos $\beta$-lactâmicos e aminoglicosídeos (CRUZ et al. 2006). Entretanto, recomendase que a PRM seja realizada com um único antimicrobiano, com base na cultura e antibiograma (SANTSCHI et al. 1998).

Cuidados especiais devem ser tomados quando antimicrobianos que apresentam efeitos adversos sistêmicos, como os aminoglicosídeos, são utilizados simultaneamente por via sistêmica e PRM. Nestes casos a administração sistêmica da droga deverá ser retardada ou a concentração da droga monitorada para não ultrapassar o limite de toxicidade. Isto porque as concentrações da droga poderão aumentar substancialmente após a liberação do torniquete, sendo particularmente importante em equinos gravemente desidratados ou sistemicamente comprometidos (RUBIO-MARTíNEZ \& CRUZ 2006).

\section{Dose e número de perfusões}

O controle de infecções ortopédicas em equinos tem sido realizado com sucesso por meio da utilização de PRM com antimicrobianos (PALMER \& HOGAN 1999, CIMETTI et al. 2004,). Entretanto, a dose mais adequada dos antimicrobianos e o número de perfusões necessárias ainda não foram estabelecidos (CRUZ et al. 2006, RUBIO-MARTíNEZ \& CRUZ 2006).

Ampla variação de doses vem sendo utilizada por diversos autores em casos clínicos e em pesquisas. Em estudo realizado por SANTSCHI et al. (1998), amicacina (125-250 mg) e gentamicina (100$300 \mathrm{mg}$ ) foram utilizadas com sucesso em diferentes condições sépticas do membro em equinos, sendo os potros perfundidos com $50 \mathrm{mg}$ (amicacina ou gentamicina). MURPHEY et al. (1999) utilizando baixa dose de amicacina $(125 \mathrm{mg})$, observaram altas concentrações do fármaco nas articulações metacarpo/metatarso falangeana e interfalangeana distal. Entretanto, altas doses como $500 \mathrm{mg}$ a $2,5 \mathrm{~g}$ de amicacina (BUTT et al. 2001, CIMETTI et al. 2004, LEVINE et al. 2010) e $1000 \mathrm{mg}$ de gentamicina (WERNER et al. 2003), tem sido utilizadas.

Devido a grandes variações individuais nas concentrações teciduais de antimicrobianos administrados por perfusão regional, embora não praticável, a dose baseada no tamanho ou peso do membro foi descrita como mais adequada (BUTT et al. 2001). Um terço da dose diária sistêmica do antimicrobiano tem sido recomendado como dose geral dos antimicrobianos para PRM (RUBIO-MARTíNEZ \& CRUZ 2006). As variações de doses descritas por vários autores para diferentes antimicrobianos em casos clínicos e pesquisas estão apresentadas na Tabela 1.

O número de perfusões necessárias para a resolução da infecção não foi estabelecido (RUBIOMARTÍNEZ \& CRUZ 2006). Com base em trabalhos realizados por diversos autores (PALMER \& HOGAN 1999, CIMETTI et al. 2004, RUBIO-MARTÍNEZ \& CRUZ 2006), uma a nove perfusões, realizadas a cada 24 horas ou a cada dois a três dias são necessárias. A PRM repetida a cada 36 horas tem sido indicada com base em estudo realizado em equinos sadios (MATTSON et al. 2004). No entanto, o tempo de eliminação de amicacina foi menor em articulações inflamadas em comparação com articulações sadias (TAINTOR et al. 2006). Além disso, o ambiente ácido e o acúmulo de debris celulares diminuem a atividade de alguns antimicrobianos (TACK \& SABATH 1985, SVENSSON et al. 1997). Em estudo retrospectivo realizado por RUBIO-MARTÍNEZ et al. (2012), uma série de cinco perfusões realizadas em dias consecutivos foi realizada independentemente do antimicrobiano inicialmente utilizado, mas em casos de infecções severas e casos refratários, as perfusões foram procedidas por longo período (até 19 perfusões), ou outra série de cinco perfusões foi repetida. No caso de infecção de estruturas sinoviais, a realização de análise citológica seriada do líquido sinovial, a partir do local infectado, é tipicamente utilizada como guia para determinar se o tratamento deve ser continuado (RUBIO-MARTíNEZ et al. 2012).

\section{Volume e infusão da solução}

Uma solução do antimicrobiano diluído em $\mathrm{NaCl} 0,9 \%$ ou Ringer com lactato é, de maneira geral, utilizada para PRM (WERNER et al. 2003, RUBIO-MARTÍNEZ et al. 2005, LEVINE et al. 2010). O volume ideal da solução ainda não foi determinado (RUBIO-MARTíNEZ \& CRUZ 2006, CRUZ et al. 2006), no entanto, o volume de $60 \mathrm{~mL}$ é frequentemente utilizado, observando-se adequada distribuição do fármaco (SANTSCHI et al. 1998, MURPHEY et al. 1999, ALKABES et al. 2011). Volumes de $20 \mathrm{~mL}$ (WERNER et al. 2003), $50 \mathrm{~mL}$ (LEVINE et al. 2010), $100 \mathrm{~mL}$ (KELMER et al. 2009) e $250 \mathrm{~mL}$ (CIMETTI et al. 2004) foram descritos para PRM em equinos adultos. Entretanto, para potros, recomenda-se o uso de volumes menores, 
variando entre 10 e $35 \mathrm{~mL}$ (RUBIO-MARTíNEZ \& CRUZ 2006).

Em estudo realizado por MATTSON et al. (2004), os autores determinaram que o volume de $0,1 \mathrm{~mL} / \mathrm{kg}$, baseado no peso corporal do animal, seria mais adequado, visto que diminuiria variações devido a capacidade individual da vasculatura do membro. HYDE et al. (2013) observaram concentrações elevadas $(125,9 \mu \mathrm{g} / \mathrm{mL}$ ) de gentamicina (dose $500 \mathrm{mg}$ ) no líquido sinovial da articulação metacarpofalangeana após perfusão de baixo volume de solução $(10 \mathrm{ml})$, utilizando a veia digital com torniquete aplicado distal ao carpo. Os autores não evidenciaram influência do volume de perfusão sobre a concentração do antimicrobiano no líquido sinovial quando comparam a perfusão de $10 \mathrm{~mL}, 30 \mathrm{~mL}(82,7 \mu \mathrm{g} / \mathrm{mL})$ e $60 \mathrm{~mL}$ $(56,1 \mu \mathrm{g} / \mathrm{mL})$. Como regra geral, quanto maior o volume utilizado, maior a pressão intravascular alcançada e, consequentemente, maior será a taxa de difusão da droga para os tecidos. Por outro lado, há maior risco de vazamento do antimicrobiano, sob o torniquete para a circulação sistêmica, em casos onde a pressão intravascular é exacerbadamente elevada (RUBIO-MARTíNEZ \& CRUZ 2006).

A administração da solução poderá ser realizada em bolus manual (BUTT et al. 2001), ou por meio de bombas de infusão (RUBIO-MARTíNEZ et al. 2005). No entanto, devido as altas pressões medulares alcançadas durante a infusão intraóssea (até 450 psi), a utilização de bombas de infusão torna-se inviável para esta modalidade (RUBIO-MARTíNEZ et al. 2005). Neste caso, infusão manual com seringas de pequeno volume $(10 \mathrm{~mL}$ ) permite maior controle da infusão (BUTT et al. 2001). Taxas de infusão de $2 \mathrm{~mL} / \mathrm{min}$. tem sido utilizadas (RUBIO-MARTÍNEZ et al. 2005) com bombas de infusão. Manualmente, a infusão tem sido realizada durante o período de 1 a 12 minutos (MURPHEY et al. 1999, BUTT et al. 2001, LEVINE et al. 2010). Para a utilização de vancomicina recomenda-se que a taxa de infusão seja limitada a $2 \mathrm{~mL} / \mathrm{min}$. a uma concentração máxima de $5 \mathrm{mg} / \mathrm{mL}$, devido ao potencial risco de tromboflebite (CRUZ et al. 2006).

Tabela 1. Antimicrobianos e variação de doses descritos por diferentes autores para perfusão regional do membro em equinos.

Table 1. Antimicrobials and dose variation described by different authors for regional limb perfusion in horses.

\begin{tabular}{|c|c|c|}
\hline Antimicrobiano & Dose & Referência \\
\hline Amicacina & $125 \mathrm{mg}$ a $2,5 \mathrm{~g}$ & $\begin{array}{l}\text { MURPHEY et al. (1999), BUTT et al. (2001), CIMETTI et al. } \\
\text { (2004), ERRICO et al. (2008), LEVINE et al. (2010), RUBIO- } \\
\text { MARTíNEZ et al. (2012), KELMER et al. (2012a) }\end{array}$ \\
\hline Ampicilina & $9 \mathrm{~g}$ & CRUZ et al. (2006) \\
\hline Cefazolina & 1 a $2 \mathrm{~g}$ & RUBIO-MARTÍNEZ et al. (2012) \\
\hline Ceftiofur sódico & 1 a $2 \mathrm{~g}$ & $\begin{array}{l}\text { PILLE et al. (2005), KELMER et al. (2012b), RUBIO- } \\
\text { MARTíNEZ et al. (2012) }\end{array}$ \\
\hline Cefotaxima & $125 \mathrm{mg}$ a $1 \mathrm{~g}$ & SWINEBROAD et al. (2003), KELMER et al. (2012b) \\
\hline Cloranfenicol & 1 a $2 \mathrm{~g}$ & KELMER et al. (2012b), KELMER et al. (2014) \\
\hline Enrofloxacina & $1,5 \mathrm{mg} / \mathrm{kg} ; 1,0 \mathrm{~g}$ & PARRA-SANCHEZ et al. (2006), KELMER et al. (2012b) \\
\hline Eritromicina & $1 \mathrm{~g}^{*}$ & KELMER et al. (2009) \\
\hline Gentamicina & $100 \mathrm{mg}$ a $1 \mathrm{~g} ; 2,2 \mathrm{mg} / \mathrm{kg}$ & $\begin{array}{l}\text { WHITEHAIR (1992), SANTSCHI et al. (1998), PALMER \& } \\
\text { HOGAN (1999), WERNER et al. (2003), MATTSON et al. } \\
\text { (2004), RUBIO-MARTÍNEZ et al. (2012), HYDE et al. } \\
\text { (2013) }\end{array}$ \\
\hline Imipeném & $500 \mathrm{mg}$ & $\begin{array}{l}\text { KELMER et al. (2012b), KELMER et al. (2009), KELMER et } \\
\text { al. (2017) }\end{array}$ \\
\hline Penicilina Potássica & $10^{6} \mathrm{UI}$ & PALMER \& HOGAN (1999) \\
\hline Ticarcilina & $125 \mathrm{mg}$ a $1,7 \mathrm{~g}$ & KELMER et al. (2012b) \\
\hline Vancomicina & $300 \mathrm{mg}$ a $1 \mathrm{~g}$ & $\begin{array}{l}\text { RUBIO-MARTíNEZ et al. (2005), RUBIO-MARTíNEZ et al. } \\
\text { (2006), KELMER et al. (2012b) }\end{array}$ \\
\hline
\end{tabular}

*Dose utilizada em potro para o tratamento de artrite séptica por Rhodococcus equi. 


\section{Aplicação clínica}

A técnica de PRM com antimicrobianos em equinos é considerada viável e clinicamente segura, e pode ser facilmente aplicada como técnica complementar no tratamento de infecções ortopédicas (RUBIOMARTíNEZ et al. 2012). Os métodos de PRM promovem altas concentrações de antimicrobianos em estruturas da região distal do membro quando comparado com administração sistêmica (MURPHEY et al. 1999, BUTT et al. 2001). Isto é possível devido ao alto gradiente de concentração e pressão obtidos entre os compartimentos intravascular e extravascular durante a perfusão, permitindo a difusão do agente antimicrobiano em tecidos pouco perfundidos (FINSTERBUSCH \& WEINBERG 1972). A PRM leva a concentrações elevadas do antimicrobiano nos tecidos alvo, com risco diminuído para o desenvolvimento de efeitos adversos sistêmicos e custo reduzido (LEVINE et al. 2010). A técnica é indicada para combater infecções ortopédicas envolvendo ossos, articulações, bainhas sinoviais e tecidos moles dos membros de equinos. Entretanto, indica-se que PRM com antimicrobianos seja utilizada como técnica complementar a outras terapias, incluindo terapia antibiótica sistêmica, anti-inflamatória, debridamento cirúrgico, lavagem articular, repouso entre outros (RUBIO-MARTíNEZ \& CRUZ 2006, RUBIO-MARTÍNEZ et al. 2012).

Em estudos que avaliaram o tratamento de diferentes condições sépticas do membro utilizando PRM com antimicrobianos associada a outras terapias, as taxas de sobrevivência variaram de $72 \%$ a $92 \%$ (SANTSCHI et al. 1998, PALMER \& HOGAN 1999, CIMETTI et al. 2004, RUBIO-MARTÍNEZ et al. 2012). CIMETTI et al. (2004) encontraram taxa de sobrevivência de $89 \%$ (16/18) utilizando PRM com amicacina (1000-2000 mg) e DMSO (250 mL sol. $20 \%$ ), associado a outras modalidades de tratamento, sendo que 13 animais retornaram à função atlética. Em estudo retrospectivo realizado por RUBIO-MARTíNEZ et al. (2012), onde a PRM com diferentes antimicrobianos foi utilizada como complemento a outras modalidades de tratamento, $72 \%$ (67/96) dos equinos do grupo portador de infecções sinoviais receberam alta hospitalar e, 53\% (73/96) daquele sem que informações foram disponíveis, sobreviveram no período igual ou maior que três meses. Informações relacionadas ao retorno à atividade prévia foram disponíveis em 35 casos, nos quais foi observado retorno com nível de desempenho igual ou superior ao anterior em $80 \%(28 / 35)$ dos animais. Os equinos do grupo portador de lacerações (incluindo estruturas sinoviais) receberam alta hospitalar em 94\% (47/50) dos casos e, 92\% (34/37) daqueles em que informações foram disponíveis, sobreviveram no período igual ou maior que três meses. O retorno a atividade prévia em nível de desempenho igual ou superior ao anterior foi reportado em $73 \%$ (24/33) dos casos em que as informações foram disponíveis.

A avaliação da PRM com antimicrobianos torna-se difícil, visto que geralmente é utilizada como complemento ao tratamento convencional, incluindo a administração sistêmica de antimicrobianos, antiinflamatórios, lavagem articular, debridamento cirúrgico e repouso (SANTSCHI et al. 1998, RUBIOMARTÍNEZ \& CRUZ 2006, RUBIO-MARTÍNEZ et al. 2012). Entretanto, as altas concentrações de antimicrobianos alcançadas nos tecidos (WERNER et al. 2003, PILLE et al. 2005, RUBIO-MARTíNEZ et al. 2005) e a resposta clínica bem sucedida em uma grande variedade de casos clínicos em que a PRM com antimicrobianos foi utilizada (SANTSCHI et al. 1998, CIMETTI et al. 2004, RUBIO-MARTíNEZ et al. 2012), suportam a eficácia da técnica.

\section{CONCLUSÃO}

Conclui-se que a utilização das modalidades de PRM com antimicrobianos são práticas seguras, de fácil realização e baixo custo. As altas concentrações de antimicrobianos alcançados no local da infecção são responsáveis pela eficácia da técnica, juntamente a outras terapias. Apesar de aspectos como dose, número de perfusões e volume da solução ideais ainda não serem determinados, diversos estudos demonstram que a técnica é altamente eficaz no tratamento das infecções ortopédicas. Dessa forma, PRM com antimicrobianos pode ser utilizada como terapia complementar ao tratamento em equinos acometidos por processos infecciosos dos membros.

\section{REFERÊNCIAS}

ABRAHAMSEN E et al. 1989. Tourniquet-induced hypertension in a horse. Journal of the American Veterinary Medical Association 194: 386-388.

ALKABES SB et al. 2011. Comparison of two tourniquets and determination of amikacin sulfate concentrations after metacarpophalangeal joint lavage performed simultaneously with intravenous regional limb perfusion in horses. American Journal of Veterinary Research 72: 613-619.

BUTT TD et al. 2001. Comparison of 2 techniques for regional antibiotic delivery to the equine forelimb: intraosseous perfusion vs. intravenous perfusion. Canadian Veterinary Journal 42: 617-622.

CIMETTI LJ et al. 2004. How to perform intravenous regional limb perfusion using amikacin and DMSO. In: 50 Annual 
Convention of the American Association of Equine Practitioners. Proceedings... Denver: International Veterinary Information Service. p. 219-223.

COPLAND VS et al. 1989. Blood pressure response to tourniquet use in anesthetized horses. Journal of the American Veterinary Medical Association 195: 1097-1103.

CRUZ AM et al. 2006. New antimicrobials, systemic distribution, and local methods of antimicrobials delivery in horses. Veterinary Clinics Equine Practice 22: 297-322.

ERRICO JA et al. 2008. Comparison of two indirect techniques for local delivery of a high dose of an antimicrobial in the distal portion of forelimbs of horses. American Journal of Veterinary Research 69: 334-342.

FINSTERBUSCH A \& WEINBERG H. 1972. Venous perfusion of the limb with antibiotics for osteomyelitis and other chronic infections. The Journal of Bone \& Joint Surgery 54: 1227-1234.

HARTMANN FA et al. 1997. Isolation of methicillin-resistant Staphylococcus aureus from a postoperative wound infection in a horse. Journal of the American Veterinary Medical Association 211: 590-592.

HYDE RM et al. 2013. The influence of perfusate volume on antimicrobial concentration in synovial fluid following intravenous regional limb perfusion in the standing horse. The Canadian Veterinary Journal 54: 363-367.

KELMER G et al. 2009. Evaluation of indwelling intravenous catheters for the regional perfusion of the limbs of horses. The Veterinary Record 165: 496-501.

KELMER G et al. 2012a. Evaluation of regional limb perfusion with amikacin using the saphenous, cephalic, and palmar digital veins. Journal of Veterinary Pharmacology and Therapeutics 36: 236-240.

KELMER G et al. 2012b. Indwelling cephalic or saphenous vein catheter use for regional limb perfusion in 44 horses with synovial injury involving the distal aspect of the limb. Veterinary Surgery 41: 938-943.

KELMER G et al. 2014. Evaluation of regional limb perfusion with chloramphenicol using the saphenous of cephalic vein in standing horses. Journal of Veterinary Pharmacology and Therapeutics 38: 35-40.

KELMER G et al. 2017. Evaluation of the pharmacokinetics of imipenem following regional limb perfusion using the saphenous and the cephalic veins in standing horses. Research in Veterinary Science 114: 64-68.

LEVINE DG et al. 2010. Efficacy of three tourniquet types for intravenous antimicrobial regional limb perfusion in standing horses. Veterinary Surgery 39: 1021-1024.

LUGO J. 2009. Regional Limb Perfusion with Antimicrobials. In: ROBINSON NE \& SPRAYBERRY KA. Robinson's Current Therapy in Equine Medicine. 6.ed. Saint Louis: Elsevier. p. 529-530.

LUGO J. 2015. Managing Orthopedic Infections. In: SPRAYBERRY KA \& ROBINSON NE. Robinson's Current Therapy in Equine Medicine. 7.ed. Saint Louis: Elsevier. p. 793-797.

MATTSON S et al. 2004. Intraosseous gentamicin perfusion of the distal metacarpus in standing horses. Veterinary Surgery 33: 180-186.

MOORE RM et al. 1992. Antimicrobial susceptibility of bacterial isolates from 233 horses with musculoskeletal infection during 1979-1989. Equine Veterinary Journal 24: 450-456.

MURPHEY ED et al. 1999. Regional intravenous perfusion of the distal limb of horses with amikacin sulfate. Journal Veterinary Pharmacology and Therapeutics 22: 68-71.

NAVARRE CB et al. 1999. Ceftiofur distribution in plasma and joint fluid following regional limb injection in cattle. Journal Veterinary Pharmacology and Therapeutics 22: 13-19.

PALMER SE \& HOGAN PM. 1999. How to perform Regional limb perfusion in the standing horse. In: 45 Annual Meeting of the American Association of Equine Practitioners. Proceedings... Kentucky. p. 124-127.

PARKER RA et al. 2010. Osteomyelitis and osteonecrosis after intraosseous perfusion with gentamicin. Veterinary Surgery 39: 644-648.

PARRA-SANCHEZ A et al. 2006. Pharmacokinetics and pharmacodynamics of enrofloxacin and low dose of amikacin administered via regional intravenous limb perfusion in standing horses. American Journal of Veterinary Research 67: 1687-1695.

PILLE F et al. 2005. Synovial fluid and plasma concentrations of ceftiofur after regional intravenous perfusion in the horse. Veterinary Surgery 34: 610-617.

RAFAEL LA et al. 2014. Avaliação do efeito trombogênico da perfusão regional intravenosa com gentamicina em equinos. Arquivo Brasileiro de Medicina Veterinária e Zootecnia 66: 1449-1456.

RICHARDSON DW \& AHERN BJ. 2012. Sinovial and Osseous Infections. In: AUER JA \& STICK JA. Equine Surgery. 4.ed. Saint Louis: Elsevier. p. 1189-1200.

RUBIO-MARTÍNEZ LM \& CRUZ AM. 2006. Antimicrobial regional limb perfusion in horses. Journal of the American Veterinary Medical Association 228: 706-712.

RUBIO-MARTÍNEZ LM et al. 2005. Medullary plasma pharmacokinetics of vancomycin after intravenous and intraosseous perfusion of the proximal phalanx in horses. Veterinary Surgery 34: 618-624.

RUBIO-MARTíNEZ LM et al. 2006. Evaluation of safety and pharmacokinetics of vancomycin after intraosseous regional limb perfusion and comparison of results with those obtained after intravenous regional limb perfusion in horses. American Journal of Veterinary Research 67: 1701-1707.

RUBIO-MARTÍNEZ LM et al. 2012. Clinical use of antimicrobial regional limb perfusion in horses: 174 cases (19992009). Journal of the American Veterinary Medical Association 241: 1650-1658.

SANTSCHI EM et al. 1998. How to perform equine intravenous digital perfusion. In: 44 Annual Convention of the American Association of Equine Practitioners. Proceedings... Baltimore. p. 124-127.

SCHNEIDER RK et al. 1992. A retrospective study of 192 horses affected with septic arthritis/tenosynovitis. Equine 
Veterinary Journal 24: 436-442.

SCHNEIDER RK. 2006. Synovial and Osseous Infections. In: AUER, JA \& STICK JA. Equine Surgery. 3.ed. Saint Louis: Elsevier. p. 1121-1129.

SISSON S \& GROSSMAN JD. 1986. Anatomia dos animais domésticos. 5.ed. Rio de Janeiro: Guanabara Koogan. $1134 p$.

SVENSSON E et al. 1997. Pharmacodynamic effects of antibiotics and antibiotic combinations on growing and nongrowing Staphylococcus epidermidis cells. Antimicrobial Agents and Chemotherapy 41: 107-111.

SWINEBROAD EL et al. 2003. Osteomyelitis secondary to trauma involving the proximal end of the radius in horse: five cases (1987-2001). Journal of the American Veterinary Medical Association 223: 486-491.

TACK KJ \& SABATH LD. 1985. Increased minimum inhibitory concentrations with anaerobiasis for tobramycin, gentamicin, and amikacin, compared to latamoxef, piperacillin, chloramphenicol, and clindamycin. Chemotherapy 31 : 204-210.

TAINTOR $\mathrm{J}$ et al. 2006. Comparison of amikacin concentrations in normal and inflamed joints of horses following intraarticular administration. Equine Veterinary Journal 38: 189-191.

WERNER LA et al. 2003. Bone gentamicin concentrations after intra-articular injections or regional intravenous perfusion in the horse. Veterinary Surgery 32: 559-565.

WHITEHAIR KJ et al. 1992. Regional limb perfusion for antibiotic treatment of experimentally induced septic arthritis. Veterinary Surgery $21: 367-373$. 